\title{
Crystal Morphology Modeling of Solvates and Hydrates of Organic Molecular Crystals: Olanzapine Solvate and Dihydrate Supporting information
}

\author{
Yuanyuan Sun, ${ }^{1,2}$, Susan M. Reutzel-Edens ${ }^{3}$, Rajni M. Bhardwaj ${ }^{3,4}$, Michael F. Doherty ${ }^{2, *}$ \\ ${ }^{1}$ College of Chemistry \& Chemical Engineering, Yangzhou University, Yangzhou 225002, P. R. China. \\ ${ }^{2}$ Department of Chemical Engineering, University of California, Santa Barbara, CA 93106, United States. \\ ${ }^{3}$ Eli Lilly and Company, Indianapolis, Indiana 46285, United States. \\ ${ }^{4}$ Pharmaceutical Research Laboratories, AbbVie, North Chicago, IL 60064, USA.
}




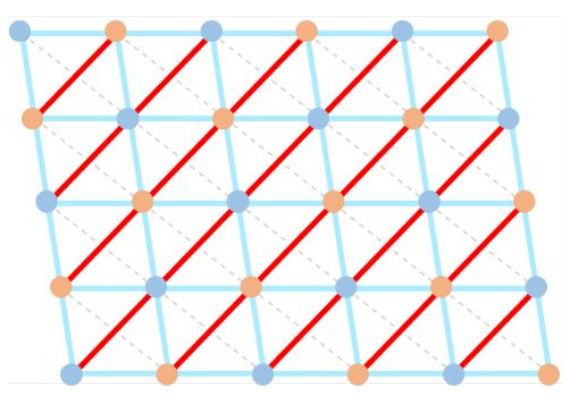

(a)

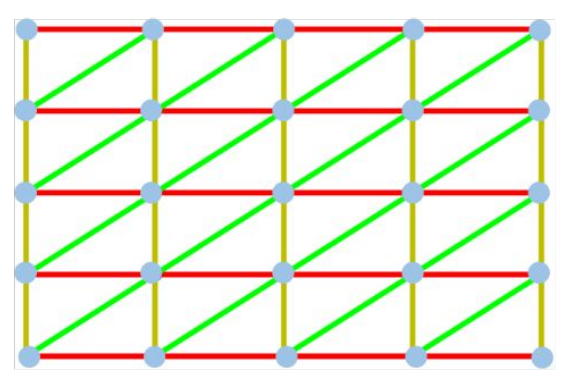

(d)

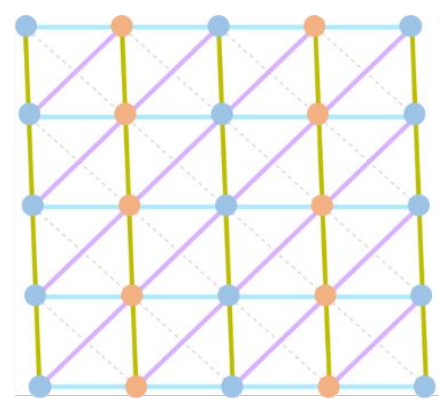

(b)

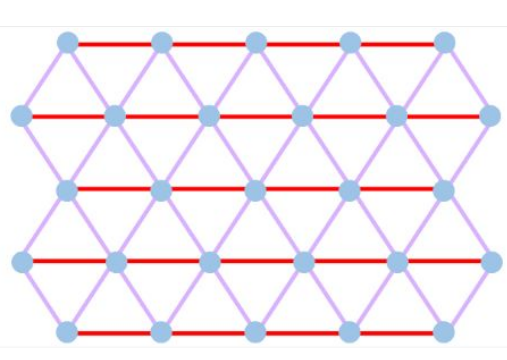

(e)

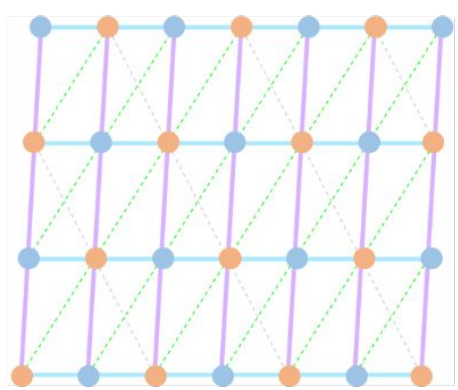

(c)

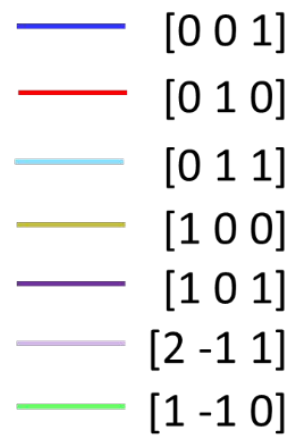

Figure S1. Bonding structures of different F-faces for tetramer growth unit with OZPN-methanol binding energy $-5.51 \mathrm{kcal} / \mathrm{mol}$ (a) $\left\{\begin{array}{lll}1 & 0 & 0\end{array}\right\}$ face, (b) $\left\{\begin{array}{llll}0 & 1 & 1\end{array}\right\}$ face, (c) $\left\{\begin{array}{lll}1 & 1 & \overline{1}\end{array}\right\}$ face, (d) $\left\{\begin{array}{lll}0 & 0 & 2\end{array}\right\}$ face, and (e) $\left\{\begin{array}{lll}1 & 0 & \overline{2}\end{array}\right\}$ face 


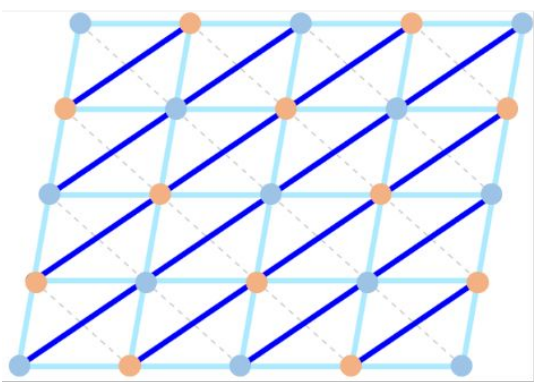

(a)

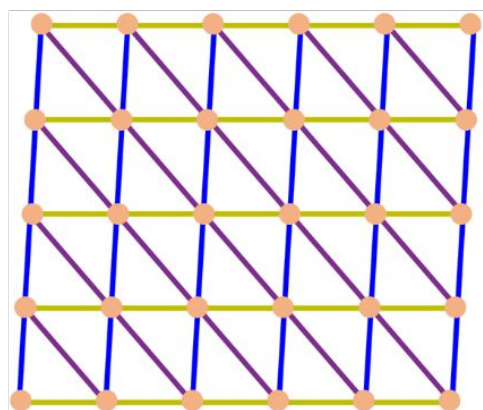

(d)

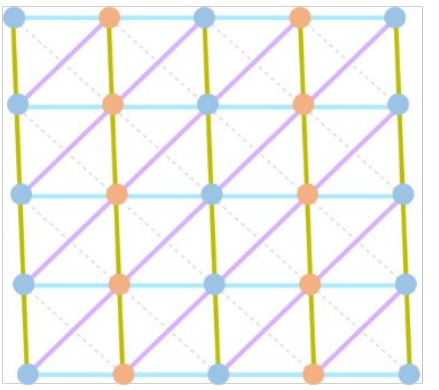

(b)

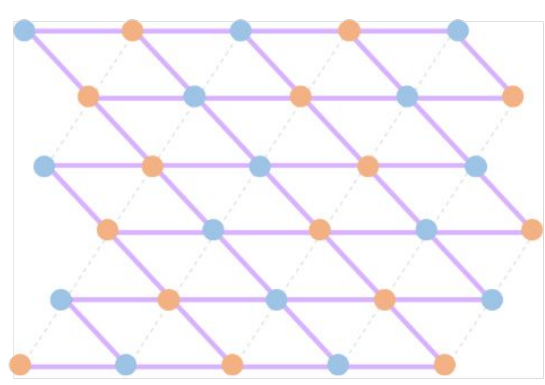

(e)

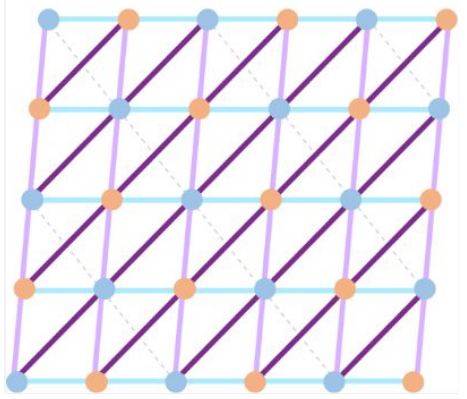

(c)

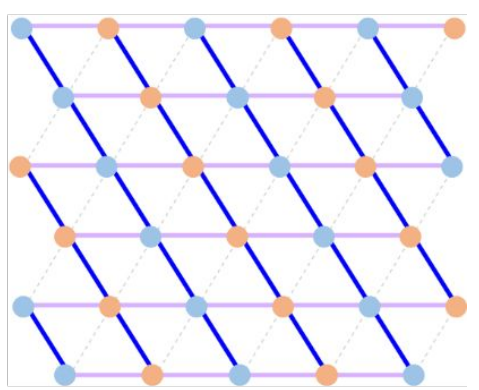

(f)

Figure S2. Bonding structures of different F-faces for tetramer growth unit with OZPN-methanol binding energy $-7.08 \mathrm{kcal} / \mathrm{mol}$ (a) $\left\{\begin{array}{lll}1 & 0 & 0\end{array}\right\}$ face, (b) $\left\{\begin{array}{lll}0 & 1 & 1\end{array}\right\}$ face, (c) $\left\{\begin{array}{lll}1 & 1 & \overline{1}\end{array}\right\}$ face, (d) $\left\{\begin{array}{lll}0 & 2 & 0\end{array}\right\}$ face, (e) $\left\{\begin{array}{lll}1 & 0 & \overline{2}\end{array}\right\}$ face, and $\left\{\begin{array}{lll}1 & 2 & 0\end{array}\right\}$ face 


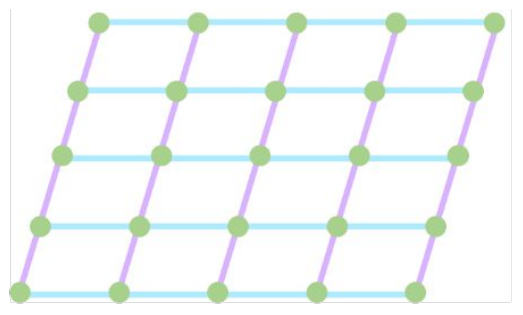

(a)

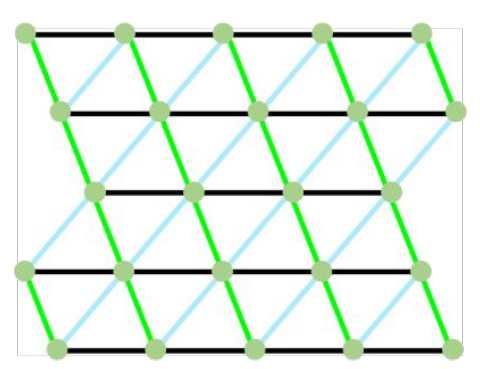

(d)

(g)

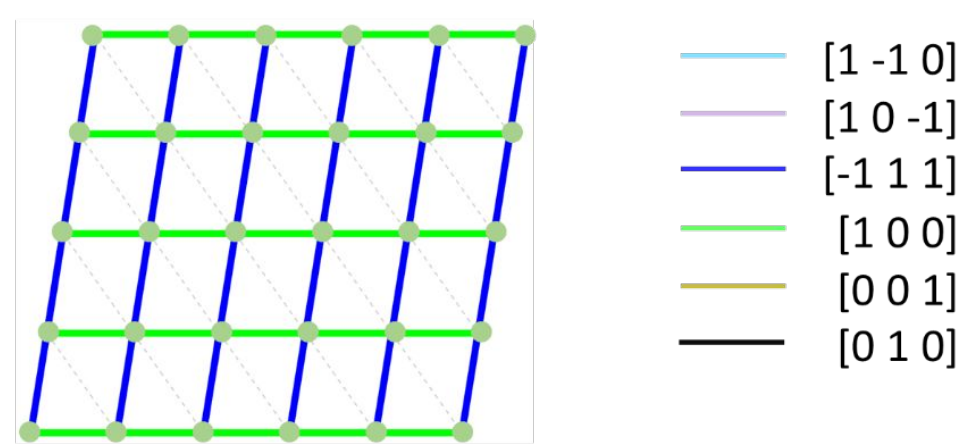

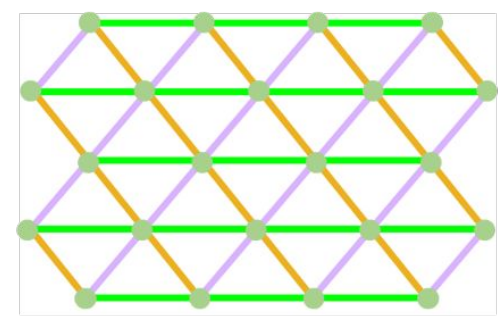

(b)

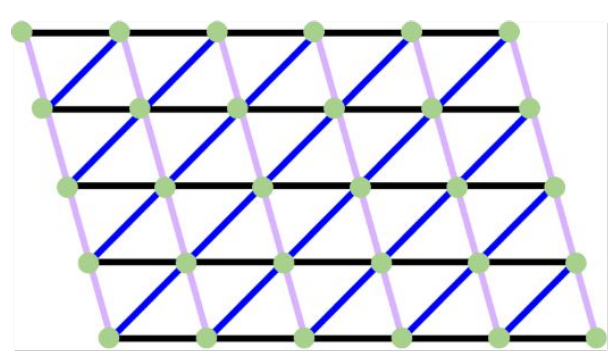

(e)

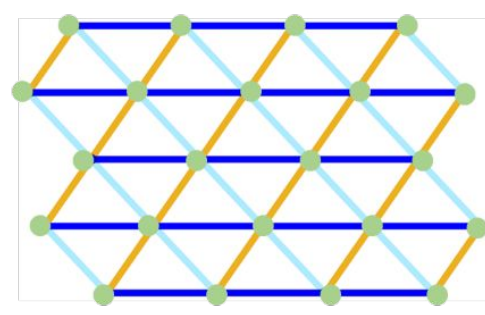

(c)

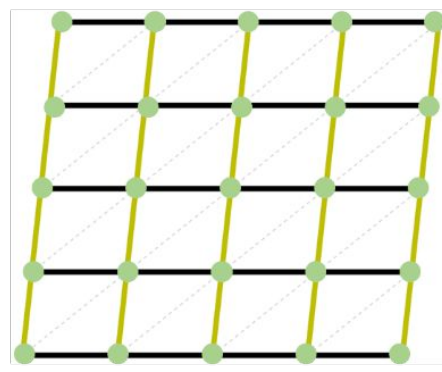

(f)

Figure S3. Bonding structures of different F-faces for OZPN-water dihydrate (a) $\left\{\begin{array}{lll}1 & 1 & 1\end{array}\right\}$ face, (b) $\left\{\begin{array}{lll}0 & 1 & 0\end{array}\right\}$ face, (c) $\left\{\begin{array}{lll}1 & 1 & 0\end{array}\right\}$ face, (d) $\left\{\begin{array}{lll}0 & 0 & 1\end{array}\right\}$ face, (e) $\left\{\begin{array}{lll}1 & 0 & 1\end{array}\right\}$ face, (f) $\left\{\begin{array}{lll}1 & 0 & 0\end{array}\right\}$ face, and (g) $\left\{\begin{array}{lll}0 & 1 & \overline{1}\end{array}\right\}$ face 


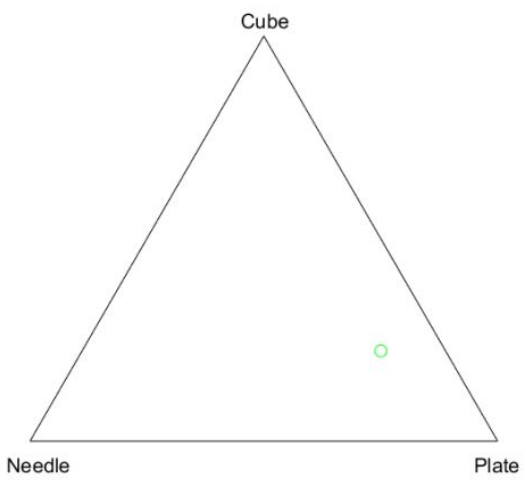

\# 1: methanol, T: $273.00 \mathrm{~K}$ S: 1.10 , soli \# 2: methanol, T: $273.00 \mathrm{~K}, \mathrm{~S}: 1.14$, sol \# 3: methanol, T: $273.00 \mathrm{~K}, \mathrm{~S}: 1.17$, sol

\# 4: methanol, T: $273.00 \mathrm{~K}, \mathrm{~S}: 1.21$, sol \# 5: methanol, T: $273.00 \mathrm{~K}, \mathrm{~S}: 1.25$, sol \# 6: methanol, T: $273.00 \mathrm{~K}, \mathrm{~S}: 1.29$, sol \# 7: methanol, T: $273.00 \mathrm{~K}, \mathrm{~S}: 1.33$, sol \# 8: methanol, T: $273.00 \mathrm{~K}, \mathrm{~S}: 1.37$, sol A: : methanol, T: $273.00 \mathrm{~K}$ S: 1.41 , sol \# 10: methanol, T: $273.00 \mathrm{~K}, \mathrm{~S}: 1.46$, sc \# 11: methanol, T: $273.00 \mathrm{~K}, \mathrm{~S}: 1.51$, sc \# 12: methanol, T: $273.00 \mathrm{~K}, \mathrm{~S}: 1.55$, sc \# 13: methanol, T: $273.00 \mathrm{~K}, \mathrm{~S}: 1.60, \mathrm{sc}$ \# 14: methanol, T: $273.00 \mathrm{~K}$ S: 1.66 sc \# 15: methanol, T: $273.00 \mathrm{~K}$ s: 1.71 , \#15: methanol, T: $273.00 K$, S: 1.71 , sc \# 16: methanol, T: $273.00 K, \mathrm{~S}: 1.76$, sc \# 17: methanol, T: $273.00 \mathrm{~K}, \mathrm{~S}: 1.82$, sc \# 18: methanol, T: $273.00 \mathrm{~K}, \mathrm{~S}: 1.88$, sc \# 19: methanol, T: $273.00 \mathrm{~K}, \mathrm{~S}: 1.94$, sc

\# 20: methanol, T: $273.00 \mathrm{~K}$ S: 2.00 , sc

Solvent: methanol

T: $273.00 \mathrm{~K}$

S: 1.100000

Solvent Model: vOCG

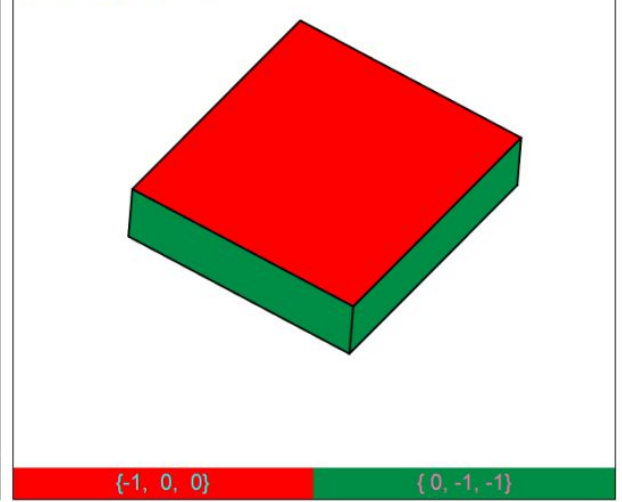

(a) Supersaturation sweep for olanzapine-methanol solvate

Supersaturation Sweep Result

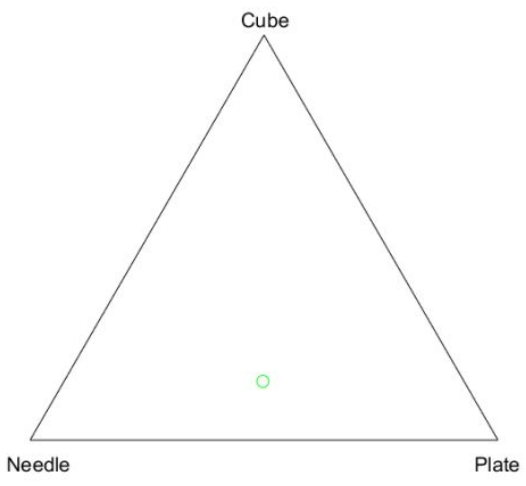

\# 1: water, T: $273.00 \mathrm{~K}, \mathrm{~S}: 1.10$, solven \# 2: water, T: $273.00 \mathrm{~K}$, S: 1.14 , solvent \# 3: water, T: $273.00 \mathrm{~K}, \mathrm{~S}$ : 1.17 , solven $\mathrm{T}$ : $273.00 \mathrm{~K}, \mathrm{~S}: 1.21$, solvent \# 5: water, T: $273.00 \mathrm{~K}, \mathrm{~S}: 1.25$, solvent \# 6: water, T: $273.00 \mathrm{~K}, \mathrm{~S}: 1.29$, solvent \# 7: water, T: $273.00 \mathrm{~K}, \mathrm{~S}: 1.33$, solvent \# 8: water, T: $273.00 \mathrm{~K}, \mathrm{~S}: 1.37$, solvent \# 9: water, T: $273.00 \mathrm{~K}, \mathrm{~S}: 1.41$, solvent \# 10: water, T: $273.00 \mathrm{~K}, \mathrm{~S}: 1.46$, solve \# 11: water, T: $273.00 \mathrm{~K}$ S: 1.51 , solve \# 12: water, T: $273.00 \mathrm{~K}, \mathrm{~S}: 1.55$, solve \# 13: water, T: $273.00 \mathrm{~K}, \mathrm{~S}: 1.60$, solvel \# 14: water, T: $273.00 \mathrm{~K}, \mathrm{~S}: 1.66$, solve \# 15: water, T: $273.00 \mathrm{~K}, \mathrm{~S}: 1.71$, solve \# 16: water, T: $273.00 \mathrm{~K}, \mathrm{~S}: 1.76$, solvel \# 17: water, T: $273.00 \mathrm{~K}$, S: 1.82 , solve \# 18: water, T: $273.00 \mathrm{~K}, \mathrm{~S}: 1.88$, solve \# 19: water, T: $273.00 \mathrm{~K}, \mathrm{~S}: 1.94$, solve \# 20: water, T: $273.00 \mathrm{~K}, \mathrm{~S}: 2.00$, solve

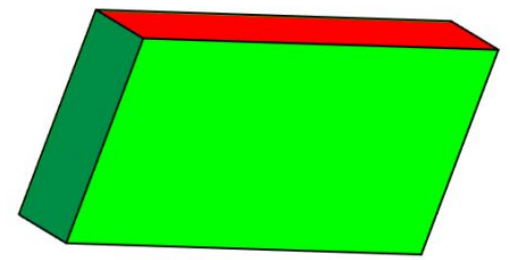

(b) Supersaturation sweep for olanzapine-water solvate

Figure S4. Supersaturation sweep for olanzapine-methanol and olanzapine-water solvates ${ }^{1}$ 
Table S1. Detailed results: tetramer growth unit with OZPN-methanol binding energy -5.51 $\mathrm{kcal} / \mathrm{mol}$ for olanzapine-methanol solvate. $\Phi$ is the kink energy; $a_{P}$ is the propagation length of adding a row of growth units; $a_{E}$ is the width of a growth units along the edge; $v$ is the forward velocity of successive steps; $l c$ is the critical lengths of an edge; $h$ is the step height; and $R$ is the normal growth rate of a face.

\begin{tabular}{|c|c|c|c|c|c|c|c|c|}
\hline Face & Edge & $\Phi(\mathrm{kcal} / \mathrm{mol})$ & $a_{P}(\AA)$ & $\overline{a_{E}(\AA)}$ & $v(\tilde{A} / \mathrm{s})$ & lc $(\AA)$ & $h(\AA)$ & $R$ \\
\hline \multirow{3}{*}{$\begin{array}{lll}1 & 0 & 0\end{array}$} & {$[010]$} & -5.85 & 7.14 & 12.44 & $1.10 \mathrm{E}-49$ & 24693.17 & \multirow{3}{*}{10.20} & \multirow{3}{*}{1.00} \\
\hline & [011] & -9.59 & 9.38 & 9.47 & $2.60 \mathrm{E}-52$ & 30817.22 & & \\
\hline & {$\left[\begin{array}{ll}01 & 1\end{array}\right]$} & -9.59 & 9.38 & 9.47 & 2.60E-52 & 30816.18 & & \\
\hline \multirow{3}{*}{$\begin{array}{lll}0 & 1 & 1\end{array}$} & {$[100]$} & -5.93 & 9.46 & 10.21 & $1.26 \mathrm{E}-49$ & 20549.01 & \multirow{3}{*}{9.38} & \multirow{3}{*}{2.96} \\
\hline & {$[2 \overline{1} 1]$} & -3.88 & 7.06 & 13.68 & $3.02 \mathrm{E}-48$ & 18003.65 & & \\
\hline & {$\left[\begin{array}{lll}0 & \overline{1} & 1\end{array}\right]$} & -9.59 & 10.20 & 9.47 & 2.83E-52 & 30817.22 & & \\
\hline \multirow{2}{*}{$\begin{array}{lll}1 & 1 & \overline{1}\end{array}$} & [011] & -9.59 & 13.65 & 9.47 & $3.79 \mathrm{E}-52$ & 30816.18 & \multirow{2}{*}{7.00} & \multirow{2}{*}{3.40} \\
\hline & {$[2 \overline{1} 1]$} & -3.88 & 9.45 & 13.68 & $4.05 \mathrm{E}-48$ & 18003.65 & & \\
\hline \multirow{3}{*}{$\begin{array}{lll}0 & 0 & 2\end{array}$} & {$[010]$} & -5.85 & 10.21 & 12.44 & $1.57 \mathrm{E}-49$ & 24693.17 & \multirow{3}{*}{7.13} & \multirow{3}{*}{700} \\
\hline & {$[100]$} & -5.74 & 12.44 & 10.21 & $2.29 \mathrm{E}-49$ & 19887.37 & & \\
\hline & [110] & -3.44 & 7.89 & 16.09 & 7.02E-48 & 18805.43 & & \\
\hline \multirow{3}{*}{$\begin{array}{lll}1 & 0 & \overline{2}\end{array}$} & {$[010]$} & -5.85 & 12.19 & 12.44 & $1.87 \mathrm{E}-49$ & 24693.17 & \multirow{3}{*}{5.97} & \multirow{3}{*}{1540} \\
\hline & {$[2 \overline{1} 1]$} & -3.75 & 11.08 & 13.68 & $5.86 \mathrm{E}-48$ & 17423.96 & & \\
\hline & [211] & -3.75 & 11.08 & 13.68 & $5.86 \mathrm{E}-48$ & 17423.96 & & \\
\hline
\end{tabular}


Table S2. Detailed results: dimer growth unit with OZPN-methanol binding energy $-5.51 \mathrm{kcal} / \mathrm{mol}$ for olanzapine-methanol solvate. $\Phi$ is the kink energy; $a_{P}$ is the propagation length of adding a row of growth units; $a_{E}$ is the width of a growth units along the edge; $v$ is the forward velocity of successive steps; $l c$ is the critical lengths of an edge; $h$ is the step height; and $R$ is the normal growth rate of a face.

\begin{tabular}{|c|c|c|c|c|c|c|c|c|}
\hline Face & Edge & $\Phi(\mathrm{kcal} / \mathrm{mol})$ & $a_{P}(\AA)$ & $a_{E}(\AA)$ & $v(\AA / s)$ & lc $(\AA)$ & $h(\AA)$ & $R$ \\
\hline \multirow{4}{*}{$\begin{array}{lll}0 & 1 & 1\end{array}$} & {$[\overline{1} 00]$} & -2.77 & 4.73 & 10.21 & $1.36 \mathrm{E}-32$ & 9606.53 & \multirow{4}{*}{9.38} & \multirow{4}{*}{$3.12 \mathrm{E}+09$} \\
\hline & {$[01 \overline{1}]$} & -9.92 & 10.20 & 4.73 & 8.30E-34 & 15940.84 & & \\
\hline & [100] & -2.77 & 4.73 & 10.21 & 7.03E-32 & 9606.53 & & \\
\hline & {$\left[\begin{array}{ll}0 & 1 \\
1\end{array}\right]$} & -9.92 & 10.20 & 4.73 & $2.01 \mathrm{E}-33$ & 15940.84 & & \\
\hline \multirow{6}{*}{$\begin{array}{lll}1 & 0 & 0\end{array}$} & {$[021]$} & -6.90 & 6.19 & 7.17 & 6.49E-36 & 16786.82 & \multirow{6}{*}{10.19} & \multirow{6}{*}{127} \\
\hline & {$\left[\begin{array}{ll}02 & 1\end{array}\right]$} & -6.89 & 6.19 & 7.17 & $6.50 \mathrm{E}-36$ & 16781.06 & & \\
\hline & {$[00 \overline{1}]$} & -4.08 & 6.22 & 7.14 & $6.15 \mathrm{E}-41$ & 9883.32 & & \\
\hline & {$\left[\begin{array}{ll}02 & 1\end{array}\right]$} & -6.90 & 6.19 & 7.17 & $9.82 \mathrm{E}-35$ & 16786.82 & & \\
\hline & {$[0 \overline{2} 1]$} & -6.89 & 6.19 & 7.17 & 9.83E-35 & 16781.06 & & \\
\hline & {$[001]$} & -4.08 & 6.22 & 7.14 & $6.15 \mathrm{E}-41$ & 9883.32 & & \\
\hline \multirow{4}{*}{$\begin{array}{lll}1 & 0 & \overline{2}\end{array}$} & {$[0 \overline{1} 0]$} & -6.34 & 12.19 & 6.22 & $3.38 \mathrm{E}-43$ & 13377.62 & \multirow{4}{*}{5.97} & \multirow{4}{*}{1} \\
\hline & [201] & -2.79 & 6.22 & 12.19 & $7.55 \mathrm{E}-41$ & 11532.92 & & \\
\hline & [010] & -6.34 & 12.19 & 6.22 & $1.59 \mathrm{E}-41$ & 13377.62 & & \\
\hline & {$[201]$} & -2.79 & 6.22 & 12.19 & $7.43 \mathrm{E}-41$ & 11532.92 & & \\
\hline
\end{tabular}


Table S3. Detailed results: tetramer growth unit with OZPN-methanol binding energy -7.08 $\mathrm{kcal} / \mathrm{mol}$ for olanzapine-methanol solvate. $\Phi$ is the kink energy; $a_{P}$ is the propagation length of adding a row of growth units; $a_{E}$ is the width of a growth units along the edge; $v$ is the forward velocity of successive steps; $l c$ is the critical lengths of an edge; $h$ is the step height; and $R$ is the normal growth rate of a face.

\begin{tabular}{|c|c|c|c|c|c|c|c|c|}
\hline Face & Edge & $\Phi(\mathrm{kcal} / \mathrm{mol})$ & $a_{P}(\AA)$ & $a_{E}(\AA)$ & $v(\AA / s)$ & $\operatorname{lc}(\AA)$ & $h(\AA)$ & $R$ \\
\hline \multirow{3}{*}{$\begin{array}{lll}1 & 0 & 0\end{array}$} & {$[001]$} & -3.91 & 6.22 & 14.28 & $1.14 \mathrm{E}-46$ & 18951.23 & \multirow{3}{*}{10.20} & \multirow{3}{*}{1.00} \\
\hline & [011] & -9.93 & 9.38 & 9.47 & $6.67 \mathrm{E}-51$ & 31897.18 & & \\
\hline & {$\left[\begin{array}{ll}01 & 1\end{array}\right]$} & -9.93 & 9.38 & 9.47 & 6.67E-51 & 31897.47 & & \\
\hline \multirow{3}{*}{$\begin{array}{lll}0 & 1 & 1\end{array}$} & {$[100]$} & -6.12 & 9.46 & 10.21 & $4.18 \mathrm{E}-48$ & 21190.12 & \multirow{3}{*}{9.38} & \multirow{3}{*}{2.98} \\
\hline & {$\left[2 \overline{1}_{1}\right]$} & -4.10 & 7.06 & 13.68 & $9.36 \mathrm{E}-47$ & 19047.05 & & \\
\hline & {$\left[\begin{array}{lll}0 & \overline{1} & 1\end{array}\right]$} & -9.93 & 10.20 & 9.47 & 7.25E-51 & 31897.47 & & \\
\hline \multirow{3}{*}{$\begin{array}{lll}1 & 1 & \overline{1}\end{array}$} & {$[101]$} & -3.32 & 7.53 & 17.17 & $3.66 \mathrm{E}-46$ & 19375.14 & \multirow{3}{*}{7.00} & \multirow{3}{*}{3.33} \\
\hline & {$[011]$} & -9.93 & 13.65 & 9.47 & $9.71 \mathrm{E}-51$ & 31897.18 & & \\
\hline & {$[2 \overline{1} 1]$} & -4.10 & 9.45 & 13.68 & $1.25 \mathrm{E}-46$ & 19047.05 & & \\
\hline \multirow{3}{*}{$\begin{array}{lll}0 & 2 & 0\end{array}$} & {$[100]$} & -5.91 & 14.26 & 10.21 & $8.89 \mathrm{E}-48$ & 20483.86 & \multirow{3}{*}{6.22} & \multirow{3}{*}{2580} \\
\hline & [101] & -3.21 & 8.48 & 17.17 & $5.02 \mathrm{E}-46$ & 18729.37 & & \\
\hline & {$[001]$} & -3.91 & 10.20 & 14.28 & $1.87 \mathrm{E}-46$ & 18951.22 & & \\
\hline \multirow{2}{*}{$\begin{array}{lll}1 & 2 & 0\end{array}$} & [001] & -3.91 & 11.94 & 14.28 & $2.18 \mathrm{E}-46$ & 18951.23 & \multirow{2}{*}{5.31} & \multirow{2}{*}{32200} \\
\hline & {$[2 \overline{1} 1]$} & -3.96 & 12.46 & 13.68 & $2.08 \mathrm{E}-46$ & 18412.22 & & \\
\hline \multirow{2}{*}{$\begin{array}{lll}1 & 0 & \overline{2}\end{array}$} & [211] & -3.96 & 11.08 & 13.68 & $1.85 \mathrm{E}-46$ & 18412.22 & \multirow{2}{*}{5.97} & \multirow{2}{*}{34400} \\
\hline & [211] & -3.96 & 11.08 & 13.68 & $1.85 \mathrm{E}-46$ & 18412.22 & & \\
\hline
\end{tabular}


Table S4. Detailed results: dimer growth unit with OZPN-methanol binding energy $-7.08 \mathrm{kcal} / \mathrm{mol}$ for olanzapine-methanol solvate. $\Phi$ is the kink energy; $a_{P}$ is the propagation length of adding a row of growth units; $a_{E}$ is the width of a growth units along the edge; $v$ is the forward velocity of successive steps; $l c$ is the critical lengths of an edge; $h$ is the step height; and $R$ is the normal growth rate of a face.

\begin{tabular}{|c|c|c|c|c|c|c|c|c|}
\hline Face & Edge & $\Phi(\mathrm{kcal} / \mathrm{mol})$ & $a_{P}(\AA)$ & $a_{E}(\AA)$ & $v(\mathrm{~A} / \mathrm{s})$ & lc $(\AA)$ & $h(\AA)$ & $R$ \\
\hline \multirow{4}{*}{$\begin{array}{llll}0 & 1 & 1\end{array}$} & {$[100]$} & -2.76 & 4.73 & 10.21 & $2.85 \mathrm{E}-32$ & 9545.89 & \multirow{4}{*}{9.38} & \multirow{4}{*}{$3.65 \mathrm{E}+0 \mathrm{~s}$} \\
\hline & {$\left[\begin{array}{ll}0 & 1 \\
1\end{array}\right]$} & -9.95 & 10.20 & 4.73 & $5.46 \mathrm{E}-34$ & 15989.03 & & \\
\hline & {$[\overline{1} 00]$} & -2.76 & 4.73 & 10.21 & $7.35 \mathrm{E}-31$ & 9545.89 & & \\
\hline & {$\left[\begin{array}{lll}0 & 1 & 1\end{array}\right]$} & -9.95 & 10.20 & 4.73 & $7.29 \mathrm{E}-34$ & 15989.03 & & \\
\hline \multirow{4}{*}{$\begin{array}{lll}0 & 0 & 2\end{array}$} & {$[100]$} & -2.76 & 6.22 & 10.21 & $1.90 \mathrm{E}-36$ & 9546.44 & \multirow{4}{*}{7.13} & \multirow{4}{*}{213000} \\
\hline & {$\left[\begin{array}{lll}0 & 1 & 0\end{array}\right]$} & -5.69 & 10.21 & 6.22 & $2.91 \mathrm{E}-37$ & 12002.82 & & \\
\hline & {$[\overline{1} 00]$} & -2.76 & 6.22 & 10.21 & $1.90 \mathrm{E}-36$ & 9546.44 & & \\
\hline & {$[010]$} & -5.69 & 10.21 & 6.22 & $2.64 \mathrm{E}-38$ & 12002.82 & & \\
\hline \multirow{4}{*}{$\begin{array}{lll}0 & 2 & 0\end{array}$} & {$[100]$} & -2.76 & 7.13 & 10.21 & $4.69 \mathrm{E}-32$ & 9545.33 & \multirow{4}{*}{6.22} & \multirow{4}{*}{1} \\
\hline & [001] & -6.61 & 10.20 & 7.14 & $1.61 \mathrm{E}-30$ & 16010.68 & & \\
\hline & {$[\overline{100}]$} & -2.76 & 7.13 & 10.21 & $2.12 \mathrm{E}-43$ & 9545.33 & & \\
\hline & {$[00 \overline{1}]$} & -6.61 & 10.20 & 7.14 & 4.24E-31 & 16010.68 & & \\
\hline \multirow{4}{*}{$\begin{array}{lll}1 & 0 & \overline{2}\end{array}$} & {$[0 \overline{1} 0]$} & -5.69 & 12.19 & 6.22 & $3.18 \mathrm{E}-38$ & 12002.12 & \multirow{4}{*}{5.97} & \multirow{4}{*}{40900} \\
\hline & [201] & -2.74 & 6.22 & 12.19 & $7.72 \mathrm{E}-38$ & 11315.45 & & \\
\hline & {$[010]$} & -5.69 & 12.19 & 6.22 & $1.03 \mathrm{E}-38$ & 12002.12 & & \\
\hline & {$[\overline{2} 0 \overline{1}]$} & -2.74 & 6.22 & 12.19 & $7.79 \mathrm{E}-38$ & 11315.45 & & \\
\hline
\end{tabular}


Table S5. Detailed results: hexamer growth unit for olanzapine dihydrate. $\Phi$ is the kink energy; $a_{P}$ is the propagation length of adding a row of growth units; $a_{E}$ is the width of a growth units along the edge; $v$ is the forward velocity of successive steps; $l c$ is the critical lengths of an edge; $h$ is the step height; and $R$ is the normal growth rate of a face.

\begin{tabular}{|c|c|c|c|c|c|c|c|c|}
\hline Face & Edge & $\Phi(\mathrm{kcal} / \mathrm{mol})$ & $a_{P}(\AA)$ & $a_{E}(\AA)$ & $v(\AA / \mathrm{A})$ & lc $(\AA)$ & $h(\AA)$ & $R$ \\
\hline \multirow{3}{*}{$\begin{array}{lll}0 & 0 & 1\end{array}$} & {$\left[\begin{array}{ll}1 & \overline{1} \\
0\end{array}\right]$} & -1.02 & 8.14 & 11.65 & $1.88 \mathrm{E}-50$ & 4054.25 & \multirow{3}{*}{9.32} & \multirow{3}{*}{1.00} \\
\hline & {$[010]$} & -10.15 & 9.40 & 10.10 & $5.28 \mathrm{E}-57$ & 34791.43 & & \\
\hline & {$[100]$} & -9.90 & 9.56 & 9.93 & $8.25 \mathrm{E}-57$ & 33355.38 & & \\
\hline \multirow{2}{*}{$\begin{array}{lll}1 & 0 & 0\end{array}$} & [001] & -0.85 & 10.05 & 10.51 & $2.93 \mathrm{E}-50$ & 3033.51 & \multirow{2}{*}{8.36} & \multirow{2}{*}{17.40} \\
\hline & {$[010]$} & -10.15 & 10.47 & 10.10 & $5.88 \mathrm{E}-57$ & 34791.43 & & \\
\hline \multirow{3}{*}{$\begin{array}{lll}1 & 0 & 1\end{array}$} & {$\left[\begin{array}{ll}10 & 1\end{array}\right]$} & -0.46 & 9.87 & 10.64 & 4.44E-50 & 1665.54 & \multirow{3}{*}{8.42} & \multirow{3}{*}{3.29} \\
\hline & {$[111]$} & -4.53 & 8.05 & 13.04 & $6.02 \mathrm{E}-53$ & 20051.19 & & \\
\hline & [010] & -10.15 & 10.40 & 10.10 & $5.84 \mathrm{E}-57$ & 34791.43 & & \\
\hline \multirow{2}{*}{$\begin{array}{lll}1 & 1 & 1\end{array}$} & {$[1 \overline{1} 0]$} & -1.02 & 10.36 & 11.65 & $2.40 \mathrm{E}-50$ & 4054.25 & \multirow{2}{*}{7.32} & \multirow{2}{*}{54000000} \\
\hline & {$\left[\begin{array}{ll}10 & 1\end{array}\right]$} & -0.46 & 11.35 & 10.64 & $5.10 \mathrm{E}-50$ & 1665.54 & & \\
\hline \multirow{3}{*}{$\begin{array}{lll}0 & 1 & 0\end{array}$} & {$[10 \overline{1}]$} & -0.46 & 8.71 & 10.64 & $3.92 \mathrm{E}-50$ & 1665.54 & \multirow{3}{*}{9.53} & \multirow{3}{*}{30.50} \\
\hline & {$[100]$} & -9.90 & 9.34 & 9.93 & $8.06 \mathrm{E}-57$ & 33355.38 & & \\
\hline & {$[001]$} & -0.85 & 8.82 & 10.51 & $2.57 \mathrm{E}-50$ & 3033.51 & & \\
\hline \multirow{3}{*}{$\begin{array}{lll}1 & 1 & 0\end{array}$} & {$\left[\begin{array}{ll}1 & \overline{1} \\
0\end{array}\right]$} & -1.02 & 9.99 & 11.65 & $2.31 \mathrm{E}-50$ & 4054.25 & \multirow{3}{*}{7.59} & \multirow{3}{*}{172000} \\
\hline & [001] & -0.85 & 11.07 & 10.51 & $3.22 \mathrm{E}-50$ & 3033.51 & & \\
\hline & {$\left[\begin{array}{lll}1 & \overline{1} & \overline{1}\end{array}\right]$} & -4.53 & 8.93 & 13.04 & $6.68 \mathrm{E}-53$ & 20051.19 & & \\
\hline \multirow{2}{*}{$\begin{array}{lll}0 & 1 & \overline{1}\end{array}$} & {$[100]$} & -9.90 & 12.91 & 9.93 & $1.11 \mathrm{E}-56$ & 33355.38 & \multirow{2}{*}{6.90} & \multirow{2}{*}{4.14} \\
\hline & {$\left[\begin{array}{llll}1 & 1 & 1\end{array}\right]$} & -4.53 & 9.83 & 13.04 & 7.35E-53 & 20051.19 & & \\
\hline
\end{tabular}


Table S6. Detailed results: trimer growth unit for olanzapine dihydrate. $\Phi$ is the kink energy; $a_{P}$ is the propagation length of adding a row of growth units; $a_{E}$ is the width of a growth units along the edge; $v$ is the forward velocity of successive steps; $l c$ is the critical lengths of an edge; $h$ is the step height; and $R$ is the normal growth rate of a face.

\begin{tabular}{|c|c|c|c|c|c|c|c|c|}
\hline Face & Edge & $\Phi(\mathrm{kcal} / \mathrm{mol})$ & $\overline{a_{P}(\AA)}$ & $\overline{a_{E}(\AA)}$ & $v(\AA / s)$ & lc $(\AA)$ & $h(\AA)$ & $\bar{R}$ \\
\hline \multirow{6}{*}{$\begin{array}{lll}0 & 0 & 1\end{array}$} & {$\left[\begin{array}{ll}0 \overline{1} 0] \\
\end{array}\right.$} & -7.52 & 4.70 & 10.10 & $9.84 \mathrm{E}-49$ & 25777.34 & \multirow{6}{*}{9.32} & \multirow{6}{*}{9680} \\
\hline & {$[\overline{1} 00]$} & -9.57 & 9.56 & 4.96 & $8.90 \mathrm{E}-47$ & 21222.05 & & \\
\hline & {$[\overline{1} 20]$} & -5.52 & 4.89 & 9.71 & 2.43E-44 & 19840.17 & & \\
\hline & {$[010]$} & -7.52 & 4.70 & 10.10 & $8.53 \mathrm{E}-50$ & 25777.34 & & \\
\hline & {$[100]$} & -15.63 & 9.56 & 4.96 & 4.84E-46 & 21222.05 & & \\
\hline & {$[1 \overline{2} 0]$} & -6.52 & 4.89 & 9.71 & $2.80 \mathrm{E}-43$ & 19840.17 & & \\
\hline \multirow{4}{*}{$\begin{array}{lll}1 & 0 & 1\end{array}$} & {$[\overline{1} 01]$} & -2.62 & 4.94 & 10.64 & $1.01 \mathrm{E}-44$ & 9454.30 & \multirow{4}{*}{8.42} & \multirow{4}{*}{144} \\
\hline & {$[\overline{1} 11]$} & -6.18 & 4.03 & 13.04 & $7.88 \mathrm{E}-48$ & 27373.87 & & \\
\hline & {$[10 \overline{1}]$} & -2.89 & 4.94 & 10.64 & $9.17 \mathrm{E}-48$ & 10449.94 & & \\
\hline & {$[1 \overline{11}]$} & -6.18 & 4.03 & 13.04 & 4.61E-52 & 27373.88 & & \\
\hline \multirow{4}{*}{$\begin{array}{lll}1 & 1 & 1\end{array}$} & {$[1 \overline{1} 01]$} & -2.89 & 5.68 & 10.64 & $8.88 \mathrm{E}-48$ & 10450.11 & \multirow{4}{*}{7.32} & \multirow{4}{*}{9720} \\
\hline & {$[\overline{1} 10]$} & -17.22 & 10.36 & 5.83 & $8.61 \mathrm{E}-48$ & 23174.12 & & \\
\hline & {$[10 \overline{1}]$} & -2.89 & 5.68 & 10.64 & $1.15 \mathrm{E}-49$ & 10449.92 & & \\
\hline & {$[1 \overline{1} 0]$} & -17.22 & 10.36 & 5.83 & $3.15 \mathrm{E}-46$ & 23174.12 & & \\
\hline \multirow{4}{*}{$\begin{array}{lll}0 & 1 & 0\end{array}$} & {$[\overline{1} 01]$} & -2.89 & 4.36 & 10.64 & $2.16 \mathrm{E}-47$ & 10450.11 & \multirow{4}{*}{9.53} & \multirow{4}{*}{180000} \\
\hline & {$[100]$} & -15.63 & 9.34 & 4.96 & 4.73E-46 & 21222.05 & & \\
\hline & {$[10 \overline{1}]$} & -2.89 & 4.36 & 10.64 & $1.42 \mathrm{E}-48$ & 10449.92 & & \\
\hline & {$[100]$} & -9.57 & 9.34 & 4.96 & $8.74 \mathrm{E}-47$ & 21222.06 & & \\
\hline \multirow{2}{*}{$\begin{array}{lll}1 & 1 & 0\end{array}$} & [1111] & -6.78 & 4.46 & 13.04 & $1.60 \mathrm{E}-46$ & 30020.86 & \multirow{2}{*}{7.59} & \multirow{2}{*}{1} \\
\hline & {$[\overline{1} 10]$} & -16.88 & 9.99 & 5.83 & $3.04 \mathrm{E}-46$ & 23174.12 & & \\
\hline
\end{tabular}




\begin{tabular}{|c|c|c|c|c|c|c|c|c|}
\hline & {$[1 \overline{12}]$} & -3.11 & 5.65 & 10.31 & $9.47 \mathrm{E}-48$ & 17494.36 & & \\
\hline & [1]1] & -6.78 & 4.46 & 13.04 & 8.79E-54 & 30020.86 & & \\
\hline & {$[1 \overline{1} 0]$} & -6.21 & 9.99 & 5.83 & $8.98 \mathrm{E}-42$ & 23174.12 & & \\
\hline & {$[\overline{1} 12]$} & -3.11 & 5.65 & 10.31 & $9.55 \mathrm{E}-40$ & 17494.36 & & \\
\hline \multirow{4}{*}{$\begin{array}{lll}2 & 1 & 1\end{array}$} & [1111] & -6.78 & 6.94 & 13.04 & $4.66 \mathrm{E}-46$ & 30020.86 & \multirow{4}{*}{4.88} & \multirow{4}{*}{13600} \\
\hline & {$[\overline{1} 20]$} & -7.11 & 9.32 & 9.71 & $2.27 \mathrm{E}-48$ & 21622.52 & & \\
\hline & [111] & -6.78 & 6.94 & 13.04 & $3.63 \mathrm{E}-46$ & 30020.86 & & \\
\hline & {$[1 \overline{2} 0]$} & -6.01 & 9.32 & 9.71 & 7.39E-42 & 21622.52 & & \\
\hline
\end{tabular}




\section{References}

1. Landis, S.; Zhao, Y.; Doherty, M. F., Digital design of crystalline solids. Comput. Chem. Eng. 2020, 133, 106637. 